\title{
Random Walk Based Sampling for Load Balancing in Multi-Server Systems
}

\author{
Dengwang Tang \\ University of Michigan \\ Ann Arbor, Michigan, USA \\ dwtang@umich.edu
}

\author{
Vijay G. Subramanian \\ University of Michigan \\ Ann Arbor, Michigan, USA \\ vgsubram@umich.edu
}

\begin{abstract}
In multi-server systems, a classical job assignment algorithm works as follows: at the arrival of each job, pick $d$ servers independently and uniformly at random and send the job to the least loaded server among the $d$ servers. This model is known as the powerof- $d$ choices algorithm. In this paper, we analyze a variant of this algorithm, where $d$ servers are sampled through $d$ independent non-backtracking random walks on a $k$-regular graph. The random walkers are periodically reset to independent uniform random positions. Under some assumptions on the underlying graph, we show that the system dynamics under this new algorithm converges to the solution of a deterministic ordinary differential equation (ODE), which is the same ODE as the classical power-of-d choices. We also show that the new algorithm stablizes the system, and the stationary distribution of the system converges to the stationary solution of the ODE. The new scheme can be considered as a derandomized version of power-of- $d$ choices as it reduces the use of randomness while maintaining the performance of power-of-d choices.
\end{abstract}

\section{CCS CONCEPTS}

- Mathematics of computing $\rightarrow$ Probability and statistics; Discrete mathematics; • Theory of computation $\rightarrow$ Randomness, geometry and discrete structures;

\section{KEYWORDS}

Queueing Theory; Random Walks; Load Balancing; Martingale Concentration Inequality; Fluid Limit Approximation

\section{ACM Reference Format:}

Dengwang Tang and Vijay G. Subramanian. 2019. Random Walk Based Sampling for Load Balancing in Multi-Server Systems. In ACM SIGMETRICS /International Conference on Measurement and Modeling of Computer Systems (SIGMETRICS '19 Abstracts), fune 24-28, 2019, Phoenix, AZ, USA. ACM, New York, NY, USA, 2 pages. https://doi.org/10.1145/3309697.3331506

\section{INTRODUCTION}

In the era of Big Data, the computing systems handling the computations typically consist of multiple servers. These computing systems are able to handle multiple jobs at the same time. As the

Permission to make digital or hard copies of part or all of this work for personal or classroom use is granted without fee provided that copies are not made or distributed for profit or commercial advantage and that copies bear this notice and the full citation on the first page. Copyrights for third-party components of this work must be honored. For all other uses, contact the owner/author(s)

SIGMETRICS '19 Abstracts, fune 24-28, 2019, Phoenix, AZ, USA

(C) 2019 Copyright held by the owner/author(s).

ACM ISBN 978-1-4503-6678-6/19/06.

https://doi.org/10.1145/3309697.3331506 scale of such systems grows larger and larger, load balancing algorithms, which determine how to route jobs to servers, require careful design. It is well known that the Join-the-Shortest-Queue (JSQ) Algorithm is optimal in several settings [4]. However, JSQ is not practical since it has a huge messaging cost. Another classical load balancing algorithm, called the power-of-d-choices algorithm $[9,10,14]$, is as follows: At each job arrival, pick $d$ servers independently and uniformly at random, and dispatch the job to the server with the shortest queue. There has been significant work on this algorithm that we briefly outline below. It was shown that, when $d \geq 2$, the queuing delay can be reduced significantly compared to the case where $d=1[7-10,14]$. Subsequent works proposed and analyzed several variants of power-of- $d$-choices model. Mitzenmacher et al. proposed a scheme where the dispatcher keeps $m$ servers in memory at the same time of probing $d$ random servers [11]. The fluid limit approximation result for this scheme is established in [6]. Yang, Srikant, and Kang [15] analyzed a variant of power-of-d-choices for batch arrivals, where slightly more than 1 servers is sampled for each task in a job. Mukherjee, Borst and Leeuwarden [12] and Budhiraja, Mukherjee, and $\mathrm{Wu}$ [3] analyzed a scheme where $d$ random servers are probed through a random vertex and its neighbors. Many variants of power-of-d-choices algorithms are also analyzed in the simplistic and static ball-in-bins model, where the maximum load, or the number of balls in the fullest bin, is analyzed. See [13] for a list of references.

In many of the schemes described above, the sampling is via independent uniformly random variables, which uses $\Theta(\log (n))$ randomness, and hence would be considered inefficient in the computer science literature [5]. In the same literature, random walks on graphs have been suggested as an efficient way of sampling [5]. Alon et al. analyzed the number of visits a non-backtracking random walk on an high-girth expander graph [1] [2]. The result shows that a sample path of a non-backtracking random walk on certain graphs has very similar behaviour to independent random choices in terms of statistics in a time window of certain length, while simple random walks do not share this property. With this as inspiration, in this paper we will analyze a variant of powerof-d-choices algorithm based on random walks on graphs. More specifically, based on our recent work in [13], in which three variants of power-of- $d$-choices are analyzed in balls-in-bins model, we seek to investigate the performance of these schemes in queuing system models. With the dynamic nature of the queueing model owing to service completions, in contrast to the static setting of the balls-in-bins model, it is not clear whether the dependent sampling of random walks will still yield good performance. One of the schemes proposed in [13] is as follows: assuming that $n$ servers are connected through a high-girth $k$-regular graph $G, d$ bins are 
sampled using $d$ independent non-backtracking random walks on $G$, where the random walkers are reset to independent uniform random positions periodically. We will call this scheme the NonBacktracking Random Walk with Restart based Power-of-d-choices (NBRWR-Pod) algorithm. In this paper, we analyze the NBRWRPod algorithm in the standard low traffic queuing system setting of load $\rho<1$. The NBRWR-Po $d$ algorithm can be considered as a derandomization of power-of- $d$ algorithm, since it achieves the same performance of power-of-d choices while using less randomness.

The performance characterization of the NBRWR-Po $d$ algorithm is presented via the following results:

(1) We analyze the NBRWR-Pod algorithm with the method of fluid-limit approximation. We show that the system dynamics for a large-scale multi-server system up to any finite time $T>0$ can be approximated by the solution to a deterministic ordinary differential equation (ODE), which is the same ODE for the regular power-of-d choices.

(2) We show that for any finite $n$, the NBRWR-Pod algorithm stabilizes the system.

(3) We show that the stationary distribution of the queue lengths under NBRWR-Pod algorithm converges to the stationary solution of the limiting differential equation in $L_{1}$.

\section{MODEL}

We consider following standard queuing system model: jobs arrives according to a Poisson process with rate $\lambda n$ where $\lambda<1$ is a constant. There are $n$ servers/queues in the system. At the time of an arrival, the dispatcher samples the queues of a subset of servers and dispatches the arrival to one of the sampled queues to wait for service. The service time for each job at each server is i.i.d. exponentially distributed with mean 1 . The reset period for the NBRWR-Pod scheme is set to be $\lfloor c \log n\rfloor$, where $c$ is a positive constant.

In order for the NBRWR-Pod dispatching scheme to perform well, we need the following assumption on the graph $G$ :

Assumption 1 ( $G$ is of high girth). The girth of the graph $G$ is greater than or equal to $2\left\lceil\alpha \log _{k-1} n\right\rceil+1$ for some constant $\alpha>0$.

\section{MAIN RESULTS}

The following results are established under the above model and NBRWR-Pod scheme.

Theorem 1. Consider the dynamic system $\mathbf{x}(t) \in[0,1]^{\mathbb{Z}_{+}} d e-$ scribed by the following differential equations:

$$
\begin{aligned}
\frac{\mathrm{d} x_{i}}{\mathrm{~d} t} & =\lambda\left(x_{i-1}^{d}-x_{i}^{d}\right)-\left(x_{i}-x_{i+1}\right) \quad i \geq 1 \\
x_{0}(t) & \equiv 1
\end{aligned}
$$

Let $\mathrm{X}^{(n)}(t)=\left(X_{i}^{(n)}(t)\right)_{i \in \mathbb{Z}_{+}}$be an infinite dimensional vector, where $X_{i}^{(n)}(t)$ is the proportion of queues with length exceeding (or equal to) $i$ at time $t$. Suppose that $(a) \mathrm{X}^{(n)}(0)$ is deterministic; (b) $\lim _{n \rightarrow \infty}\left\|\mathbf{X}^{(n)}(0)-\mathbf{x}(0)\right\|_{1}=0 ;(c)\|\mathbf{x}(0)\|_{1}<+\infty$, then for every finite $\stackrel{n \rightarrow \infty}{T>0}$

$$
\lim _{n \rightarrow \infty} \sup _{0 \leq t \leq T}\left\|\mathbf{X}^{(n)}(t)-\mathbf{x}(t)\right\|_{1}=0 \quad \text { a.s. }
$$

Theorem 2. Let $Q_{j}^{(n)}(t)$ be the length of queue $j$ at time t. For any initial queue length $\mathrm{Q}^{(n)}(0), \mathrm{Q}^{(n)}(t) \stackrel{t \rightarrow \infty}{\Longrightarrow} \hat{\mathbf{Q}}^{(n)}$ for some random vector $\hat{\mathrm{Q}}^{(n)}$.

Theorem 3. $\lim _{n \rightarrow \infty} \mathrm{E}\left[\left\|\hat{\mathbf{X}}^{(n)}-\hat{\mathbf{x}}\right\|_{1}\right]=0$, where $\hat{\mathbf{x}}$ is the unique fixed point of (1).

Given the theorem, we can characterize the expected queuing delay under NBRWR-Pod.

COROLlary 1. The average waiting time for a job in a stationary system of $n$ servers converges to $\sum_{i=1}^{\infty} \lambda^{\frac{d^{i}-d}{d-1}}$ as $n$ goes to infinity.

\section{CONCLUSIONS}

In this paper we analyzed the performance of a new load balancing algorithm for the balls-in-bins problem proposed in [13], in the context of multi-server queuing systems. The new algorithm is a variant of the classical power-of-d choices algorithm, which replaces uniform independent sampling with random walks on $k$-regular graph based sampling. We show that this new algorithm yields a similar behavior as power-of-d choices for large scale systems. Hence, the new algorithm can be considered as a derandomization of power-of-d choices.

\section{REFERENCES}

[1] Noga Alon, Itai Benjamini, Eyal Lubetzky, and Sasha Sodin. 2007. Nonbacktracking random walks mix faster. Communications in Contemporary Mathematics 9, 04 (2007), 585-603.

[2] Noga Alon and Eyal Lubetzky. 2009. Poisson approximation for non-backtracking random walks. Israel fournal of Mathematics 174, 1 (2009), 227-252.

[3] Amarjit Budhiraja, Debankur Mukherjee, and Ruoyu Wu. 2017. Supermarket model on graphs. arXiv preprint arXiv:1712.07607 (2017).

[4] G. Foschini and Jack Salz. 1978. A basic dynamic routing problem and diffusion. IEEE Transactions on Communications 26, 3 (1978), 320-327.

[5] Shlomo Hoory, Nathan Linial, and Avi Wigderson. 2006. Expander graphs and their applications. Bull. Amer. Math. Soc. 43, 4 (2006), 439-561.

[6] MJ Luczak and JR Norris. 2013. Averaging over fast variables in the fluid limit for Markov chains: application to the supermarket model with memory. The Annals of Applied Probability 23, 3 (2013), 957-986.

[7] Malwina J Luczak and Colin McDiarmid. 2006. On the maximum queue length in the supermarket model. The Annals of Probability 34, 2 (2006), 493-527.

[8] Malwina J Luczak and James Norris. 2005. Strong approximation for the supermarket model. The Annals of Applied Probability 15, 3 (2005), 2038-2061.

[9] Michael Mitzenmacher. 1996. Load balancing and density dependent jump Markov processes. In IEEE Symposium on Foundations of Computer Science (FOCS). IEEE, 213.

[10] Michael Mitzenmacher. 2001. The power of two choices in randomized load balancing. IEEE Transactions on Parallel and Distributed Systems 12, 10 (2001), 1094-1104.

[11] Michael Mitzenmacher, Balaji Prabhakar, and Devavrat Shah. 2002. Load balancing with memory. In IEEE Symposium on Foundations of Computer Science (FOCS). IEEE, 799.

[12] Debankur Mukherjee, Sem C Borst, and Johan SH van Leeuwaarden. 2018. Asymptotically optimal load balancing topologies. Proceedings of the ACM on Measurement and Analysis of Computing Systems 2, 1 (2018), 14.

[13] Dengwang Tang and Vijay G. Subramanian. 2018. Balanced Allocation with Random Walk Based Sampling. arXiv preprint arXiv:1810.02722 (2018).

[14] Nikita Dmitrievna Vvedenskaya, Roland L'vovich Dobrushin, and Fridrikh Izrailevich Karpelevich. 1996. Queueing system with selection of the shortest of two queues: An asymptotic approach. Problemy Peredachi Informatsii 32, 1 (1996), $20-34$.

[15] Lei Ying, R. Srikant, and Xiaohan Kang. 2015. The power of slightly more than one sample in randomized load balancing. In Computer Communications (INFOCOM), 2015 IEEE Conference on. IEEE, 1131-1139. 